\title{
EDITORIAL
}

\section{BIOMARKERS OF AGING}

Currently THERE is a great clamoring from vocal segments of the gerontological research community, as well as from public and private funding agencies, for bigger and better biomarker research in gerontology. The time has come to raise a precautionary, even dissenting voice.

What is meant by the phrase, biomarkers of aging? In my experience, this phrase has different meanings to different people. Some regard it as a means to measure the degree of functional age as opposed to chronological age. Some regard it as a means to ascertain a rate of aging. Others regard it as a standard by which to assess the effectiveness of a therapeutic intervention. Still others regard it as a means to document successful aging.

In my opinion, history will regard research related to the identification of such biomarkers of aging as no more than of fleeting significance. Sophisticated research entails so much more than pure empiricism. The significance of research on biomarkers of aging, in my opinion, will be reserved primarily for the identification, fundamental understanding, and ability to manipulate the expression of one or more steps of a genetically determined, developmental sequence of biological events which herald the onset of altered susceptibility to environmental challenge and, ultimately, death. Indeed, the seemingly endless passion for characterizing and modifying features of the elderly inevitably will give way to the pursuit of underlying mechanisms.

Why is the practical significance of this latter approach so minimally addressed on the research agenda of the gerontological research community and that of relevant funding agencies? Why, just as cancer research passed through its long period of cure-testing and so forth, must gerontological research be doomed to repeat such mistakes? There is obvious value in the ability to distinguish between functional and chronological age. For example, why should an individual who loses one kind of mental and/or physical capability, while retaining others, be disregarded wastefully? Similar kinds of arguments may be posed with compassion as they relate to relative rates of aging, to establishment of therapeutic interventions, and to hallmarks of successful aging. After all, even I desire for my loved ones and myself a successful aging that is free of disease. ripe with continued opportunity and productivity, and complete with assurances of dignity and respect.

However, in a scholarly sense, such emotionally fashionable issues are both superficial and unsophisticated. Moreover, they tend to obscure the importance of gaining a basic understanding of the processes of aging. Continuous efforts to characterize descriptively, as well as to solve the problems of old age, at least in my opinion, should not take precedence over the pursuit of new knowledge regarding the mechanisms of aging. 
Stop-gap measures may indeed have immediate impact. This is precisely why they are too often of such great appeal to the leadership of Congress, to certain public and private funding agencies, and to others untrained in basic science. However, in the long run such stop-gap measures only perpetuate the conditions they were intended to alleviate.

What substantiation do I provide for my allegations of superficiality and lack of scholarly sophistication in current tendencies of biomarker research? In an effort to make my point, consider the following single specific example. Let us assume arbitrarily that high circulating levels of catecholamines represent one example of a biomarker of aging.

Is it possible that the high circulating level of catecholamines during aging can be utilized either itself or as one of a battery of tests in order to distinguish between chronological and functional age of the experimental subjects? Is it possible that the high circulating level of catecholamines during aging can be utilized either itself or as one of a battery of tests, when detected in the same group of individuals at two or more different points in their lifespan, to ascertain a rate of aging? Is it possible that perturbation of the high circulating level of catecholamines during aging can be utilized either itself or as one of a battery of tests to assess the efficacy of a putative therapeutic regimen? Is it possible to interpret a successful therapeutic restoration of circulating levels of catecholamines in older people to levels which are more characteristic of younger people as successful intervention, thereby restoring the individuals in question to something approaching more successful aging? In my opinion, the correct answer to each of these questions is as follows. Yes, it certainly is possible, although highly unlikely.

Why might circulating levels of catecholamines be higher in older individuals than in younger individuals? Let me identify only a few of the underlying mechanisms which conceivably might contribute to such a phenomenon of aging: 1) desensitization of catecholamine receptors on the cell membranes of target tissues; 2) diminished metabolic turnover of catecholamines; 3 ) enhanced biosynthesis and/or secretion of catecholamines; 4) one or more disease states to which the autonomic nervous system responds by a chronically enhanced outpouring of catecholamines; 5) reduced availability of essential social support systems, thereby provoking a chronic stress response in the older individuals in question; 6) an age-associated personality change which manifests itself in part as a chronic stress response; 7) an age-associated increase in the distrust of the individuals who are responsible for physical measurement of the biomarker in question; etc., etc., etc.

Given two older individuals of the identical chronological age, each with vastly different circulating levels of catecholamines, is one functionally older than the other? Is one aging more successfully than the other? Will a therapeutic intervention which successfully restores catecholamines to a lower circulating level in any manner alleviate the relevant pathology, personality change, unavailable social support, etc., etc., etc.? I have great doubts!

What might one constructively conclude from the foregoing? I have no intention whatsoever of attempting to criticize any kind of research, as long as the research is of high quality. To those of you who prefer to distinguish between functional and chronological age, to determine rates of aging, to establish successful therapeutic regimens, to characterize successful aging, that certainly is your prerogative as independent investigators. 
However, in my opinion the emphasis of gerontological research, as well as its ultimate societal impact, lies in a totally different direction. When, how and why aging begins are the more important questions. The power and beauty of basic science eventually dominates the scene in every sophisticated research community. It is only a matter of time until the biomarker of maturation of the gerontological research community will be identified as the inclination and ability of the majority of researchers and funding agencies alike to comprehend the significance and to emphasize the pursuit of underlying mechanisms. Furthermore, it is a too often forgotten lesson of the history of science that the most significant societal advances usually result not by administrative design of the funding agencies, but instead, serendipitously from straightforward, nontargeted, investigator-initiated, high quality basic research.

Richard C. Adelman

Director, Institute of Gerontology

The University of Michigan

Ann Arbor, MI 